\title{
The Medical Science Research and Development Supported by the Korea Science and Engineering Foundation
}

This study examined ways of promoting research in the medical sciences by evaluating trends in research funding, and the present status of research funding by the Korea Science and Engineering Foundation (KOSEF). This study analyzed statistics from KOSEF from 1978 to 2003 to examine support for research. In medical science field, group-based programs receive more funding than do individual-based programs. The proportion of research funds allocated to the medical sciences has increased markedly each year. Researchers in the medical sciences have submitted more articles to Science Citation Index (SCl) journals than to non-SCI journals, relative to other fields. Researchers supported by the Mission-Oriented Basic Grants program have published the majority of these papers, followed by those supported by the Programs for Leading Scientists, Regional Scientists, Leading Women Scientists, Young Scientists, and Promising Women Scientists, in that order. Funding by KOSEF reflects many decades of government support for research and development, the development and maintenance of necessary infrastructure, and the education and training of medical scientists.

Key Words : Research Support; Research; Biomedical Research; Research Activities; Korea

\author{
Tae-Sun Min, Jin Han*, \\ Seong-Yong Kim ${ }^{\dagger}$, Byoung-Doo Rhee, \\ Myung-Suk Kim ${ }^{\S}$
}

Korea Science and Engineering Foundation, Daejeon Mitochondrial Signaling Laboratory*, Department of

Physiology and Biophysics, College of Medicine, Inje University, Busan; Korea Institute of Geoscience and Mineral Resources ${ }^{\dagger}$, Daejeon; Department of Internal Medicine ${ }^{\ddagger}$, College of Medicine, Inje University, Busan Department of Physiology ${ }^{\S}$, College of Medicine, The

Catholic University of Korea, Seoul, Korea

Received : 27 December 2004 Accepted : 4 April 2005

Address for correspondence

Tae-Sun Min, Ph.D.

Korea Science and Engineering Foundation (KOSEF),

180-1 Gajeong-dong, Yuseong-gu, Daejeon 305-350, Korea

Tel : +82.42-869-6531, Fax : +82.42-869-6641

E-mail : tsmin@kosef.re.kr

*This study was supported by the Staff Study Group Grants from the Korea Science and Engineering Foundation, Republic of Korea.

\section{INTRODUCTION}

Research and development (R\&D) can be classified into basic and applied research. Since there is little consensus on the definition of basic research, various standards have been adopted (1).

Although there is no internationally accepted definition of basic science, it is generally understood to involve the natural sciences, including some of the medical sciences. The Frascati Manual of the Organization for Economic Co-operation and Development (2) includes various definitions of basic research; the best known of these defines it as experimental and theoretical work that is conducted to obtain fundamental knowledge of observable facts and phenomena, without considering specific applications or uses. Basic research does not focus on a particular objective but on experimental and theoretical work with a view to devising new theories and extending knowledge in the basic sciences and engineering (3). The purpose of basic research is to obtain general knowledge and understanding of a certain subject, without necessarily considering specific applications. From an industrial perspective, this might be defined as not pertaining to specific, commercial knowledge, but as an enhancement of scientific knowledge (4). The importance of basic research is increasing with the development of innovative knowledge, technological innovation, and knowledge-based industries; in addition, this kind of research fosters high-level science and engineering manpower.

There has been a call for increased cooperation among industries, universities, and research institutes with regard to basic research in the medical sciences so that we can face the challenges confronting us in the 21st Century. This kind of approach requires an operating paradigm whereby academia faces no walls or boundaries, allowing internationalization and mobility among academics.

In particular, the importance of focusing university research on basic studies has increased under the National Innovation System. Appropriate research funding should be allocated to ensure commitment and achievement; in addition, the mode of funding is also important. Consequently, it is important to evaluate current research funding to universities, as funding that plays a crucial role in the medical sciences. This study evaluated research in the medical sciences through an 
analysis of funding in this field by the Korea Science and Engineering Foundation (KOSEF) by comparing funding in other research sectors over the past $26 \mathrm{yr}$ and examining the number of sector-specific research tasks and funding allocations, the age and scholarship distribution of the research manpower in this field, and the number of their publications, as well as present alternatives for developing the most appropriate research strategies and systems.

\section{MATERIALS AND METHODS}

This study analyzed statistics from the Korea Science and Engineering Foundation from 1978 to 2003 to examine support for research undertaken by individuals, including new professors, basic researchers, core-specific researchers, and by those involved in International Bank for Reconstruction and Development (IBRD) loan-assisted projects, international cooperative research projects, and group/collective studies, including Mission-Oriented Basic Grants (including highend research projects), and projects for centers of excellence. We examined support for training researchers, including scholarships for masters and doctoral candidates, overseas and domestic post-doctoral training, research infrastructure establishment projects, and operational support for special research material banks and special research information centers. The statistical data do not agree with the annual accounts and project budgets of KOSEF; the statistical analysis only included support for research projects and not support for small-scale academic activities.

Since the research management system of KOSEF does not use a consistent classification system for the areas of research supported, we classified research projects into 15 categories and compiled basic statistics on each. The categories were aging and cancer, allergy and immunology, anatomy and cell biology, biochemistry and molecular biology, clinical sciences, clinical technology and methodology, genetics, hygienic chemistry, neurosciences, pharmaceutics, pharmaceutical chemistry, physiology and pharmacology, public health and safety, and others. In many cases, it was not clear which category a research project fell into. Therefore, many projects were classified as "other". For projects involving more than two researchers, we attempted to identify the interests of the chief researcher. All research funding was rounded off to the nearest thousand won and the data for the research team were processed using internal data from KOSEF.

\section{RESULTS}

\section{Analysis of research funding trends in the medical sciences}

In Korea, real national R\&D started in 1978 with the establishment of KOSEF. National R\&D funds have grown con- tinuously and reached a record 5.28 trillion won in 2003 (5). Between 1978 and 2003, KOSEF invested 129.54 billion won in a total of 38,305 grants. Of this, 19.93 billion won went towards supporting 4,054 projects in the medical sciences. Over the period studied, the medical sciences received between 10.6 and $13.6 \%$ of the grant money each year. In 2003, KOSEF spent an estimated 4.25 billion won of its own funds on $\mathrm{R} \& \mathrm{D}$ in the medical sciences, providing $32.6 \%$ of the national total of 13.04 billion won (5).

From 1978 to 2003 , the total number of projects increased by a factor of 33.5, and the corresponding research expenses increased by a factor of 695.6. For the medical sciences, in 2003,600 projects ( $21.9 \%$ of the total) received 42.5 billion won $(18.9 \%$ of the total) in grants, whereas no medical science projects were supported in 1978 (Table 1). In general, support for the medical sciences by KOSEF relative to the total number of projects has increased steadily in terms of both the number of projects and the level of funding since 1978 .

In 1983/1984, no medical science projects received grants; this gradually increased to $14.9-24.0 \%$ of projects and $11.4-$ $20.3 \%$ of total funds for 1999-2003 (Table 1). This parallels the increase in funding for medical sciences in the United States from $23.5-23.9 \%$ for $1975-1985$ to $28.9-31.1 \%$ for 1998-2001. R\&D in the U.S.A. has contributed to innovation and growth materially. Federal R\&D funds have been espe-

Table 1. Number of research projects and expenditures in medical sciences supported by the KOSEF

(Unit: Project, Thousand Korean Won)

\begin{tabular}{|c|c|c|c|c|c|c|}
\hline \multirow[b]{2}{*}{ Year } & \multicolumn{3}{|c|}{ No. of Project } & \multicolumn{3}{|c|}{ Expenditure } \\
\hline & $\begin{array}{l}\text { Med. Sci. } \\
\text { (A) }\end{array}$ & $\begin{array}{c}\text { Total } \\
\text { (B) }\end{array}$ & $\begin{array}{l}\text { Ratio } \\
(\mathrm{A} / \mathrm{B}, \%)\end{array}$ & $\begin{array}{l}\text { Med. Sci. } \\
\text { (A) }\end{array}$ & $\begin{array}{l}\text { Total } \\
\text { (B) }\end{array}$ & $\begin{array}{c}\text { Ratio } \\
(\mathrm{A} / \mathrm{B}, \%)\end{array}$ \\
\hline 1978 & 0 & 82 & 0.0 & 0 & 322,400 & 0.0 \\
\hline 1982 & 2 & 537 & 0.4 & 10,788 & $1,632,550$ & 0.7 \\
\hline 1983 & 0 & 629 & 0.0 & 0 & $2,133,010$ & 0.0 \\
\hline 1984 & 0 & 918 & 0.0 & 0 & $3,558,565$ & 0.0 \\
\hline 1985 & 0 & 1,097 & 0.0 & 0 & $5,134,633$ & 0.0 \\
\hline 1986 & 2 & 1.461 & 0.1 & 61,500 & $9,364,947$ & 0.7 \\
\hline 1987 & 8 & 1,348 & 0.6 & 231,000 & $11,142,596$ & 2.1 \\
\hline 1988 & 8 & 1,202 & 0.7 & 242.000 & $11,485,090$ & 2.1 \\
\hline 1989 & 23 & 1,608 & 1.4 & 740,000 & $14,416,002$ & 5.1 \\
\hline 1990 & 26 & 1,637 & 1.6 & 923,000 & $17,893,409$ & 5.2 \\
\hline 1991 & 45 & 1,841 & 2.4 & $1,639,760$ & $28,492,656$ & 5.8 \\
\hline 1992 & 83 & 1,585 & 5.2 & $2,449,500$ & $37,280,340$ & 6.6 \\
\hline 1993 & 102 & 1,318 & 7.7 & $3,101,200$ & $37,564,963$ & 8.3 \\
\hline 1994 & 128 & 1,566 & 8.2 & $3,860,316$ & $48,054,043$ & 8.0 \\
\hline 1995 & 203 & 2,170 & 9.4 & $6,592,328$ & $67,033,035$ & 9.8 \\
\hline 1996 & 299 & 2,406 & 12.4 & $8,502,546$ & $85,409,824$ & 10.0 \\
\hline 1997 & 303 & 2,464 & 12.3 & $9,761,790$ & $102,699,447$ & 9.5 \\
\hline 1998 & 360 & 2,617 & 13.8 & $10,508,807$ & $106,104,444$ & 9.9 \\
\hline 1999 & 275 & 1,842 & 14.9 & $13,191,006$ & $115,946,728$ & 11.4 \\
\hline 2000 & 398 & 1,968 & 20.2 & $23,584,990$ & $147,730,307$ & 16.0 \\
\hline 2001 & 544 & 2,576 & 21.1 & $30,251,547$ & $184,250,951$ & 16.4 \\
\hline 2002 & 645 & 2,688 & 24.0 & $41,184,599$ & $203,084,546$ & 20.3 \\
\hline 2003 & 600 & 2,745 & 21.9 & $42,477,224$ & $224,271,064$ & 18.9 \\
\hline Total & 4,054 & 38,305 & 10.61 & $199,313,901$ & $1,295,408,550$ & 13.6 \\
\hline
\end{tabular}


cially important to the academic sector, which is the source of much of the nation's basic research. Federal funds constituted close to $60 \%$ of academic R\&D expenditures over the past decade. The majority of expenditures for academic R\&D in 2001 went to the life sciences, which accounted for $59 \%$ of all academic R\&D expenditures, $58 \%$ of Federal academic $\mathrm{R} \& \mathrm{D}$ expenditures, and $59 \%$ of non-Federal academic R\&D expenditures. Within the life sciences, the medical sciences accounted for about $31 \%$ of academic R\&D expenditures and the biological sciences for about $18 \%$. The next largest block of academic R\&D expenditures went to engineering, with about $15 \%$ in $2001(4,6)$.

\section{Analysis of research trends in the medical sciences by type}

We examined support for individual research projects, group/collective research projects, research infrastructure,

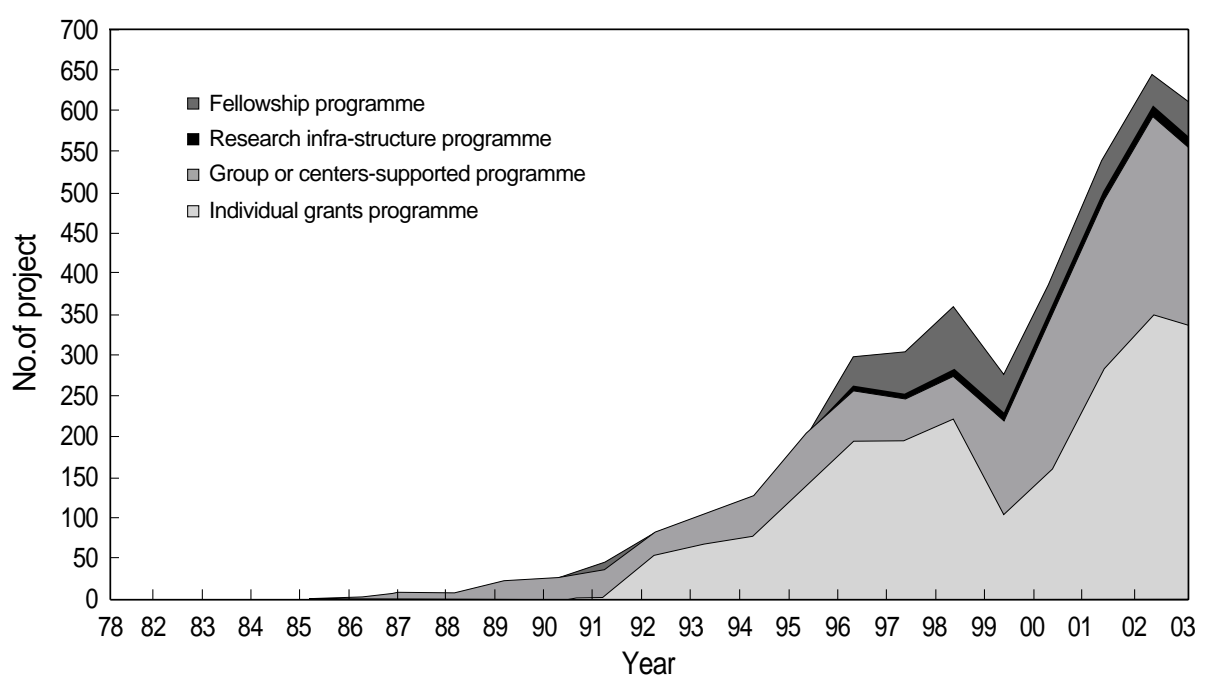

Fig. 1. Types of research programs supported by KOSEF, and the number of research projects in the medical sciences.

Table 2. The number and properties of medical research projects by funding types from KOSEF

(Unit: No. of project)

\begin{tabular}{|c|c|c|c|c|c|c|c|c|c|c|c|c|c|c|}
\hline \multirow{2}{*}{ Year } & \multicolumn{2}{|c|}{ No. of project } & \multicolumn{3}{|c|}{ Individual grants programme } & \multicolumn{3}{|c|}{$\begin{array}{c}\text { Group or centers } \\
\text {-supported programme }\end{array}$} & \multicolumn{3}{|c|}{$\begin{array}{l}\text { Research infra- } \\
\text { structure programme }\end{array}$} & \multicolumn{3}{|c|}{ Fellowship programme } \\
\hline & $\begin{array}{l}\text { Total } \\
\text { (A) }\end{array}$ & $\begin{array}{l}\text { Med. Sci. } \\
\text { (B) }\end{array}$ & $\begin{array}{l}\text { Project } \\
\text { (C) }\end{array}$ & $\begin{array}{c}\text { Ratio } \\
\text { (C/A, \%) }\end{array}$ & $\begin{array}{c}\text { Ratio } \\
(\mathrm{C} / \mathrm{B}, \%)\end{array}$ & $\begin{array}{l}\text { Project } \\
\text { (C) }\end{array}$ & $\begin{array}{c}\text { Ratio } \\
(\mathrm{C} / \mathrm{A}, \%)\end{array}$ & $\begin{array}{l}\text { Ratio } \\
(\mathrm{C} / \mathrm{B}, \%)\end{array}$ & $\begin{array}{l}\text { Project } \\
\text { (C) }\end{array}$ & $\begin{array}{l}\text { Ratio } \\
(\mathrm{C} / \mathrm{A}, \%)\end{array}$ & $\begin{array}{l}\text { Ratio } \\
(\mathrm{C} / \mathrm{B}, \%)\end{array}$ & $\begin{array}{l}\text { Project } \\
\text { (C) }\end{array}$ & $\begin{array}{l}\text { Ratio } \\
\text { (C/A, \%) }\end{array}$ & $\begin{array}{l}\text { Ratio } \\
(\mathrm{C} / \mathrm{B}, \%)\end{array}$ \\
\hline 1978 & 82 & 0 & 0 & 0.00 & 0.00 & 0 & 0.00 & 0.00 & 0 & 0.00 & 0.00 & 0 & 0.00 & 0.00 \\
\hline 1982 & 537 & 2 & 0 & 0.00 & 0.00 & 0 & 0.00 & 0.00 & 0 & 0.00 & 0.00 & 2 & 0.37 & 100.00 \\
\hline 1983 & 629 & 0 & 0 & 0.00 & 0.00 & 0 & 0.00 & 0.00 & 0 & 0.00 & 0.00 & 0 & 0.00 & 0.00 \\
\hline 1984 & 918 & 0 & 0 & 0.00 & 0.00 & 0 & 0.00 & 0.00 & 0 & 0.00 & 0.00 & 0 & 0.00 & 0.00 \\
\hline 1985 & 1,097 & 0 & 0 & 0.00 & 0.00 & 0 & 0.00 & 0.00 & 0 & 0.00 & 0.00 & 0 & 0.00 & 0.00 \\
\hline 1986 & 1,461 & 2 & 0 & 0.00 & 0.00 & 2 & 0.14 & 100.00 & 0 & 0.00 & 0.00 & 0 & 0.00 & 0.00 \\
\hline 1987 & 1,348 & 8 & 0 & 0.00 & 0.00 & 8 & 0.59 & 100.00 & 0 & 0.00 & 0.00 & 0 & 0.00 & 0.00 \\
\hline 1988 & 1,202 & 8 & 0 & 0.00 & 0.00 & 8 & 0.67 & 100.00 & 0 & 0.00 & 0.00 & 0 & 0.00 & 0.00 \\
\hline 1989 & 1,608 & 23 & 0 & 0.00 & 0.00 & 23 & 1.43 & 100.00 & 0 & 0.00 & 0.00 & 0 & 0.00 & 0.00 \\
\hline 1990 & 1,637 & 26 & 0 & 0.00 & 0.00 & 26 & 1.59 & 100.00 & 0 & 0.00 & 0.00 & 0 & 0.00 & 0.00 \\
\hline 1991 & 1,841 & 45 & 4 & 0.22 & 8.89 & 33 & 1.79 & 73.33 & 0 & 0.00 & 0.00 & 8 & 0.43 & 17.78 \\
\hline 1992 & 1,585 & 83 & 55 & 3.47 & 66.27 & 28 & 1.77 & 33.73 & 0 & 0.00 & 0.00 & 0 & 0.00 & 0.00 \\
\hline 1993 & 1,318 & 102 & 69 & 5.24 & 67.65 & 33 & 2.50 & 32.35 & 0 & 0.00 & 0.00 & 0 & 0.00 & 0.00 \\
\hline 1994 & 1,566 & 128 & 79 & 5.04 & 61.72 & 49 & 3.13 & 38.28 & 0 & 0.00 & 0.00 & 0 & 0.00 & 0.00 \\
\hline 1995 & 2,170 & 203 & 142 & 6.54 & 69.95 & 58 & 2.67 & 28.57 & 3 & 0.14 & 1.48 & 0 & 0.00 & 0.00 \\
\hline 1996 & 2,406 & 299 & 195 & 8.10 & 65.22 & 63 & 2.62 & 21.07 & 3 & 0.12 & 1.00 & 38 & 1.58 & 12.71 \\
\hline 1997 & 2,464 & 303 & 196 & 7.95 & 64.69 & 51 & 2.07 & 16.83 & 5 & 0.20 & 1.65 & 51 & 2.07 & 16.83 \\
\hline 1998 & 2,617 & 360 & 222 & 8.48 & 61.67 & 54 & 2.06 & 15.00 & 7 & 0.27 & 1.94 & 77 & 2.94 & 21.39 \\
\hline 1999 & 1,842 & 275 & 105 & 5.70 & 38.18 & 117 & 6.35 & 42.55 & 6 & 0.33 & 2.18 & 47 & 2.55 & 17.09 \\
\hline 2000 & 1,968 & 398 & 162 & 8.23 & 40.70 & 191 & 9.71 & 47.99 & 9 & 0.46 & 2.26 & 36 & 1.83 & 9.05 \\
\hline 2001 & 2,576 & 544 & 281 & 10.91 & 51.65 & 211 & 8.19 & 38.79 & 7 & 0.27 & 1.29 & 45 & 1.75 & 8.27 \\
\hline 2002 & 2,688 & 645 & 350 & 13.02 & 54.26 & 248 & 9.23 & 38.45 & 8 & 0.30 & 1.24 & 39 & 1.45 & 6.05 \\
\hline 2003 & 2,745 & 600 & 332 & 12.09 & 55.33 & 209 & 7.61 & 34.83 & 12 & 0.44 & 2.00 & 47 & 1.71 & 7.83 \\
\hline Total & 38,305 & 4,054 & 2,192 & 5.72 & 54.07 & 1,412 & 3.69 & 34.83 & 60 & 0.16 & 1.48 & 390 & 1.02 & 9.62 \\
\hline
\end{tabular}


Table 3. The medical research expenditures by funding types from KOSEF

(Unit: Thousand Korean Won)

\begin{tabular}{|c|c|c|c|c|c|c|c|c|c|c|c|c|c|c|}
\hline \multirow[b]{2}{*}{ Year } & \multicolumn{2}{|c|}{ Grant amount } & \multicolumn{3}{|c|}{$\begin{array}{l}\text { Individual grants } \\
\text { programme }\end{array}$} & \multicolumn{3}{|c|}{$\begin{array}{c}\text { Group or centers } \\
\text {-supported programme }\end{array}$} & \multicolumn{3}{|c|}{$\begin{array}{l}\text { Research infra- } \\
\text { structure programme }\end{array}$} & \multicolumn{3}{|c|}{ Fellowship programme } \\
\hline & $\begin{array}{l}\text { Total } \\
(\mathrm{A})\end{array}$ & $\begin{array}{l}\text { Med. Sci. } \\
\text { (B) }\end{array}$ & $\begin{array}{l}\text { Amount } \\
\text { (C) }\end{array}$ & $\begin{array}{c}\text { Ratio } \\
\text { (C/A, } \\
\%)\end{array}$ & $\begin{array}{c}\text { Ratio } \\
\text { (C/B, } \\
\%)\end{array}$ & $\begin{array}{l}\text { Amount } \\
\text { (C) }\end{array}$ & $\begin{array}{c}\text { Ratio } \\
(\mathrm{C} / \mathrm{A}, \\
\%)\end{array}$ & $\begin{array}{c}\text { Ratio } \\
(\mathrm{C} / \mathrm{B}, \\
\%)\end{array}$ & $\begin{array}{c}\text { Amount } \\
\text { (C) }\end{array}$ & $\begin{array}{c}\text { Ratio } \\
\text { (C/A, } \\
\%)\end{array}$ & $\begin{array}{c}\text { Ratio } \\
\text { (C/B, } \\
\%)\end{array}$ & $\begin{array}{l}\text { Amount } \\
\text { (C) }\end{array}$ & $\begin{array}{c}\text { Ratio } \\
\text { (C/A, } \\
\%)\end{array}$ & $\begin{array}{c}\text { Ratio } \\
\text { (C/B, } \\
\%)\end{array}$ \\
\hline 1978 & 322,400 & 0 & 0 & 0.00 & 0.00 & 0 & 0.00 & 0.00 & 0 & 0.00 & 0.00 & 0 & 0.00 & 0.00 \\
\hline 1982 & $1,632,550$ & 10,788 & 0 & 0.00 & 0.00 & 0 & 0.00 & 0.00 & 0 & 0.00 & 0.00 & 10,788 & 0.66 & 100.00 \\
\hline 1983 & $2,133,010$ & 0 & 0 & 0.00 & 0.00 & 0 & 0.00 & 0.0 & 0 & 0.00 & 0.00 & 0 & 0.00 & 0.00 \\
\hline 1984 & $3,558,565$ & 0 & 0 & 0.00 & 0.0 & 0 & 0.00 & 0.0 & 0 & 0.00 & 0.00 & 0 & 0.00 & 0.00 \\
\hline 1985 & $5,134,633$ & 0 & 0 & 0.00 & 0.00 & 0 & 0.00 & 0.00 & 0 & 0.00 & 0.00 & 0 & 0.00 & 0.00 \\
\hline 1986 & $9,364,947$ & 61,500 & 0 & 0.00 & 0.00 & 61,500 & 0.66 & 100.00 & 0 & 0.00 & 0.00 & 0 & 0.00 & 0.00 \\
\hline 1987 & $11,142,596$ & 231,000 & 0 & 0.00 & 0.00 & 231,000 & 2.07 & 100.00 & 0 & 0.00 & 0.00 & 0 & 0.00 & 0.00 \\
\hline 1988 & $11,485,090$ & 242,000 & 0 & 0.00 & 0.00 & 242,000 & 2.11 & 100.00 & 0 & 0.00 & 0.00 & 0 & 0.00 & 0.00 \\
\hline 1989 & $14,416,002$ & 740,000 & 0 & 0.00 & 0.00 & 740,000 & 5.13 & 100.00 & 0 & 0.00 & 0.00 & 0 & 0.00 & 0.00 \\
\hline 1990 & $17,893,409$ & 923,000 & 0 & 0.00 & 0.00 & 923,000 & 5.16 & 100.00 & 0 & 0.00 & 0.00 & 0 & 0.00 & 0.00 \\
\hline 1991 & $28,492,656$ & $1,639,760$ & 61,000 & 0.21 & 3.72 & $1,563,000$ & 5.49 & 95.32 & 0 & 0.00 & 0.00 & 15,760 & 0.06 & 0.96 \\
\hline 1992 & $37,280,340$ & $2,449,500$ & 421,000 & 1.13 & 17.19 & $2,028,500$ & 5.44 & 82.81 & 0 & 0.00 & 0.00 & 0 & 0.00 & 0.00 \\
\hline 1993 & $37,564,963$ & $3,101,200$ & 548,700 & 1.46 & 17.69 & $2,552,500$ & 6.79 & 82.31 & 0 & 0.00 & 0.00 & 0 & 0.00 & 0.00 \\
\hline 1994 & $48,054,043$ & $3,860,316$ & 879,716 & 1.83 & 22.7 & $2,980,600$ & 6.20 & 77. & 0 & 0.00 & 0.00 & 0 & 0.00 & 0.00 \\
\hline 1995 & $67,033,03$ & $6,592,328$ & $1,784,368$ & 2.66 & 27. & $4,354,960$ & 6.50 & 66. & 453,000 & 0.68 & 6.87 & 0 & 0.00 & 0.00 \\
\hline 1996 & $85,409,824$ & $8,502,546$ & $2,525,060$ & 2.96 & 29.7 & & 6.0 & & 300,000 & 0.35 & 3.5 & 541,786 & 0.63 & 6.37 \\
\hline 1997 & $102,699,447$ & $9,761,790$ & $3,067,790$ & 2.99 & 31.43 & $5,248,980$ & 5.11 & 53.77 & 580,000 & 0.56 & 5.94 & 865,020 & 0.84 & 8.86 \\
\hline 1998 & $106,104,444$ & $10,508,807$ & $3,211,120$ & 3.03 & 30.56 & $5,609,300$ & 5.29 & 53.38 & 693,000 & 0.65 & 6.59 & 995,387 & 0.94 & 9.47 \\
\hline 1999 & $115,946,728$ & $13,191,006$ & $1,502,790$ & 1.30 & 11.39 & $9,577,000$ & 8.26 & 72.60 & $1,450,000$ & 1.25 & 10.99 & 661,216 & 0.57 & 5.01 \\
\hline 2000 & $147,730,307$ & $23,584,990$ & $4,407,830$ & 2.98 & 18.69 & $17,674,979$ & 11.96 & 74.94 & 888,000 & 0.60 & 3.77 & 614,181 & 0.42 & 2.60 \\
\hline 2001 & $184,250,951$ & $30,251,547$ & $6,621,920$ & 3.59 & 21.89 & $22,231,000$ & 12.07 & 73.49 & 958,000 & 0.52 & 3.17 & 440,627 & 0.24 & 1.46 \\
\hline 2002 & $203,084,546$ & $41,184,599$ & $8,624,500$ & 4.25 & 20.94 & $30,889,000$ & 15.21 & 75.00 & 832,000 & 0.41 & 2.02 & 839,099 & 0.41 & 2.04 \\
\hline 2003 & $224,271,064$ & $42,477,224$ & $10,647,853$ & 4.75 & 25.07 & $29,917,000$ & 13.34 & 70.43 & $1,272,000$ & 0.57 & 2.99 & 640,371 & 0.29 & 1.51 \\
\hline Total & $1,465,005,550$ & $199,313,901$ & $44,303,647$ & 3.02 & 22.23 & $141,960,019$ & 9.69 & 71.22 & $7,426,000$ & 0.51 & 3.73 & $5,624,235$ & 0.38 & 2.82 \\
\hline
\end{tabular}

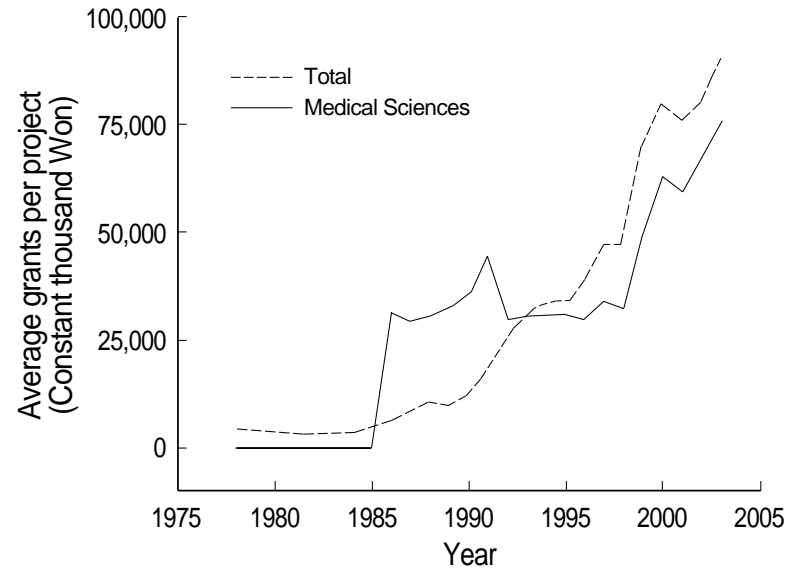

Fig. 2. Research expenditure per project in the medical sciences ( ${ }^{\star}$ Grants amounts: Datus of Individual Grants Program and Group or Centers-supported Programme).

and research manpower training. Of 4,054 programs in the medical sciences from 1978 to 2003, 2,192 (54.1\%) were individual projects, 1,412 (34.8\%) were group/collective research projects, $60(1.5 \%)$ were research infrastructure projects, and 390 (9.6\%) were manpower training projects.

Before $1990,70 \%$ of research involved group studies and few significant projects were initiated by individuals. By con-

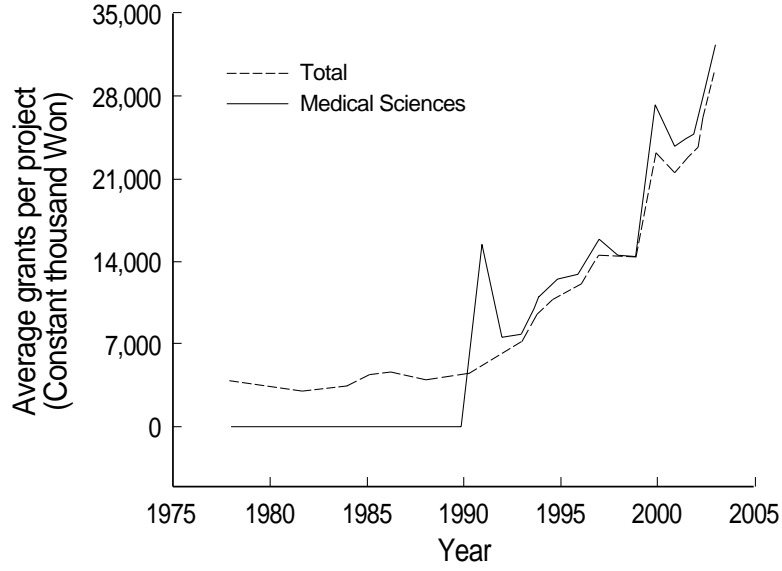

Fig. 3. Individual research grants per project in the medical sciences (Grants amounts: Datus of Individual Grants Program).

trast, since 2000 , only 35 to $48 \%$ of all medical science projects involved group or center-supported programs. In 2003, 332 grants were for individual projects ( $55 \%$ of the total for medical sciences), whereas there were no such grants before 1990 (Table 2, Fig. 1).

Between 1978 and 2003, the medical sciences received 199.3 billion won in grants out of a total of 1,465 billion won (13.6\%). Of this, 44.3 billion won $(22.2 \%)$ went to individ- 
Table 4. Manpower in medical sciences in Korea

(Unit: Headcount, \%)

\begin{tabular}{|c|c|c|c|c|c|c|c|c|c|c|c|c|c|c|c|c|c|c|c|}
\hline \multirow{2}{*}{ Sub-field } & \multirow{2}{*}{$\begin{array}{l}\text { Average } \\
\text { age (yr) }\end{array}$} & \multicolumn{2}{|c|}{ 61- } & \multicolumn{2}{|c|}{$56-60$} & \multicolumn{2}{|c|}{$51-55$} & \multicolumn{2}{|c|}{$46-50$} & \multicolumn{2}{|c|}{$41-45$} & \multicolumn{2}{|c|}{$36-40$} & \multicolumn{2}{|c|}{-35} & \multicolumn{4}{|c|}{ Total } \\
\hline & & Male & Female & Male F & Female & e Male & Female & eMale & Female & le Male & Female & e Male & Female & e Male & e Female & e Male & Female & e Total & Ratio \\
\hline Clinica Sciences & 47 & 42 & 2 & 63 & 3 & 116 & 13 & 200 & 21 & 282 & 47 & 178 & 49 & 27 & 21 & 908 & 156 & 1,064 & 23.7 \\
\hline $\begin{array}{l}\text { Clinical } \\
\text { Technology \& } \\
\text { Methodology }\end{array}$ & 48 & 10 & 10 & 13 & 19 & 29 & 27 & 48 & 56 & 55 & 44 & 26 & 20 & 5 & 15 & 186 & 191 & 377 & 8.4 \\
\hline $\begin{array}{l}\text { Anatomy \& } \\
\text { Cell Biology }\end{array}$ & 45 & 12 & 0 & 17 & 1 & 19 & 2 & 44 & 9 & 86 & 20 & 48 & 16 & 19 & 12 & 245 & 60 & 305 & 6.8 \\
\hline Aging \& Cancer & 45 & 5 & 0 & 7 & 2 & 31 & 7 & 70 & 10 & 85 & 29 & 62 & 25 & 9 & 12 & 269 & 85 & 354 & 7.9 \\
\hline $\begin{array}{l}\text { Biochemistry \& } \\
\text { Molecular Biology }\end{array}$ & 44 & 22 & 5 & 26 & 7 & 54 & 7 & 147 & 31 & 181 & 34 & 124 & 32 & 54 & 31 & 608 & 147 & 755 & 16.8 \\
\hline Genetics & 45 & 6 & 1 & 8 & 3 & 11 & 1 & 32 & 6 & 45 & 7 & 28 & 7 & 17 & 6 & 147 & 31 & 178 & 4.0 \\
\hline $\begin{array}{l}\text { Allergy \& } \\
\text { Immunology }\end{array}$ & 44 & 5 & 0 & 6 & 3 & 13 & 0 & 42 & 12 & 62 & 16 & 47 & 16 & 14 & 13 & 189 & 60 & 249 & 5.5 \\
\hline Neurosciences & 44 & 12 & 0 & 15 & 0 & 19 & 2 & 82 & 11 & 115 & 19 & 77 & 21 & 30 & 14 & 350 & 67 & 417 & 9.3 \\
\hline $\begin{array}{l}\text { Physiology \& } \\
\text { Pharmacology }\end{array}$ & 45 & 13 & 1 & 10 & 2 & 34 & 2 & 41 & 10 & 67 & 14 & 45 & 13 & 18 & 11 & 228 & 53 & 281 & 6.3 \\
\hline $\begin{array}{l}\text { Pharmaceutical } \\
\text { Chemistry }\end{array}$ & 48 & 4 & 1 & 3 & 2 & 6 & 1 & 9 & 0 & 11 & 4 & 8 & 6 & 4 & 0 & 45 & 14 & 59 & 1.3 \\
\hline Pharmacognosy & 46 & 9 & 0 & 9 & 2 & 5 & 5 & 20 & 4 & 25 & 6 & 14 & 8 & 11 & 7 & 93 & 32 & 125 & 2.8 \\
\hline Pharmaceutics & 45 & 4 & 1 & 3 & 0 & 1 & 0 & 9 & 1 & 13 & 4 & 1 & 6 & 4 & 2 & 35 & 14 & 49 & 1.1 \\
\hline Hygienic Chemistry & ry 47 & 4 & 0 & 3 & 1 & 5 & 1 & 5 & 2 & 8 & 4 & 6 & 1 & 3 & 2 & 34 & 11 & 45 & 1.0 \\
\hline $\begin{array}{l}\text { Public Health \& } \\
\text { Safety }\end{array}$ & 46 & 11 & 3 & 17 & 1 & 26 & 5 & 36 & 11 & 51 & 9 & 34 & 17 & 7 & 9 & 182 & 55 & 237 & 5.3 \\
\hline $\begin{array}{l}\text { Medical Sciences } \\
\text { Total }\end{array}$ & 46 & 159 & 24 & 200 & 46 & 369 & 73 & 785 & 184 & 1,086 & 257 & 698 & 237 & 222 & 1553 & 3,519 & 976 & 4,4951 & 100.0 \\
\hline
\end{tabular}

*Source, amended from KOSEF Manpower DB (2004).

Table 5. Output of research publications by the researchers registered in KOSEF Manpower database (Unit: Headcount, \%)

\begin{tabular}{|c|c|c|c|c|c|c|}
\hline \multirow{2}{*}{$\begin{array}{l}\text { Output of } \\
\text { research } \\
\text { publications }\end{array}$} & \multicolumn{2}{|c|}{ Non-SCI } & \multicolumn{2}{|l|}{$\mathrm{SCl}$} & \multicolumn{2}{|l|}{ Total } \\
\hline & Manpower & Ratio & Manpower & Ratio & Manpower & Ratio \\
\hline $0-5$ & 9,670 & 21.5 & 35,535 & 79.2 & 7,012 & 15.6 \\
\hline $6-10$ & 7,287 & 16.2 & 3,623 & 8.1 & 6,900 & 15.4 \\
\hline $11-20$ & 10,025 & 22.3 & 3,174 & 7.1 & 9,942 & 22.2 \\
\hline $21-30$ & 5,842 & 13.0 & 1,193 & 2.7 & 6,215 & 13.8 \\
\hline $31-40$ & 3,465 & 7.7 & 590 & 1.3 & 3,922 & 8.7 \\
\hline $41-50$ & 2,268 & 5.1 & 295 & 0.7 & 2,653 & 5.9 \\
\hline $51-60$ & 1,449 & 3.2 & 177 & 0.4 & 1,940 & 4.3 \\
\hline $61-70$ & 1,065 & 2.4 & 102 & 0.2 & 1,259 & 2.8 \\
\hline $71-$ & 3,804 & 8.5 & 186 & 0.4 & 5,032 & 11.2 \\
\hline Total & 44,875 & 100.0 & 44,875 & 100.0 & 44,875 & 100.0 \\
\hline
\end{tabular}

*Source, amended from KOSEF Manpower DB (2004).

ual research programs, 142.0 billion won $(71.2 \%)$ went to group research, 74.3 billion won $(3.7 \%)$ went to research infrastructure programs, and 56.2 billion won (2.8\%) went to manpower training programs (Table 3).

\section{Unit cost analysis of medical science research programs}

The total number of projects supported by KOSEF has increased every year, especially in the medical sciences. Research funding per project reached 90 million won in 2003.

Since 1978 the average amount of support in the medical sciences has remained low compared with other fields. Even in 2003 it amounted to only 75 million won, $83.5 \%$ of the average investment of 90 million won per other project (Fig. 2). The average amount of the Korean Research Foundation (KRF) support in the medical sciences area showed the same phenomenon, as represented by KOSEF (7).

Based on a constant price index, in the 16-yr period (19781993), the average amount granted per project to support the research programs of individuals almost doubled, from 3.9 to 7.3 million won. In the medical sciences, there are no records for before 1990, and support has gradually increased to date. This period can be regarded as a time of supporting wider fields of research. With the budget increases from the government and the implementation of the Science Research Center/Engineering Research Center (SRC/ERC), unit support has increased sharply since 1991.

This trend was a little sluggish in 1999, but improved in 2000 following both the transfer of core special research projects to the Ministry of Education (MOE) and the introduction of a new individual-support program for regional university scientists ( 25 million won), leading scientists (100 million won), and female scientists (25 million for the most promising and 40 million for excellence).

As of 2003, the unit support amount averaged 30 million won overall and 32.1 million won in the medical sciences, whereas the annual average amount per project for the period 1978 through 2003 was 20 million won for the medical 
Table 6. Output of research publications by medical science researchers registered in KOSEF Manpower DB (Unit: Headcount, \%)

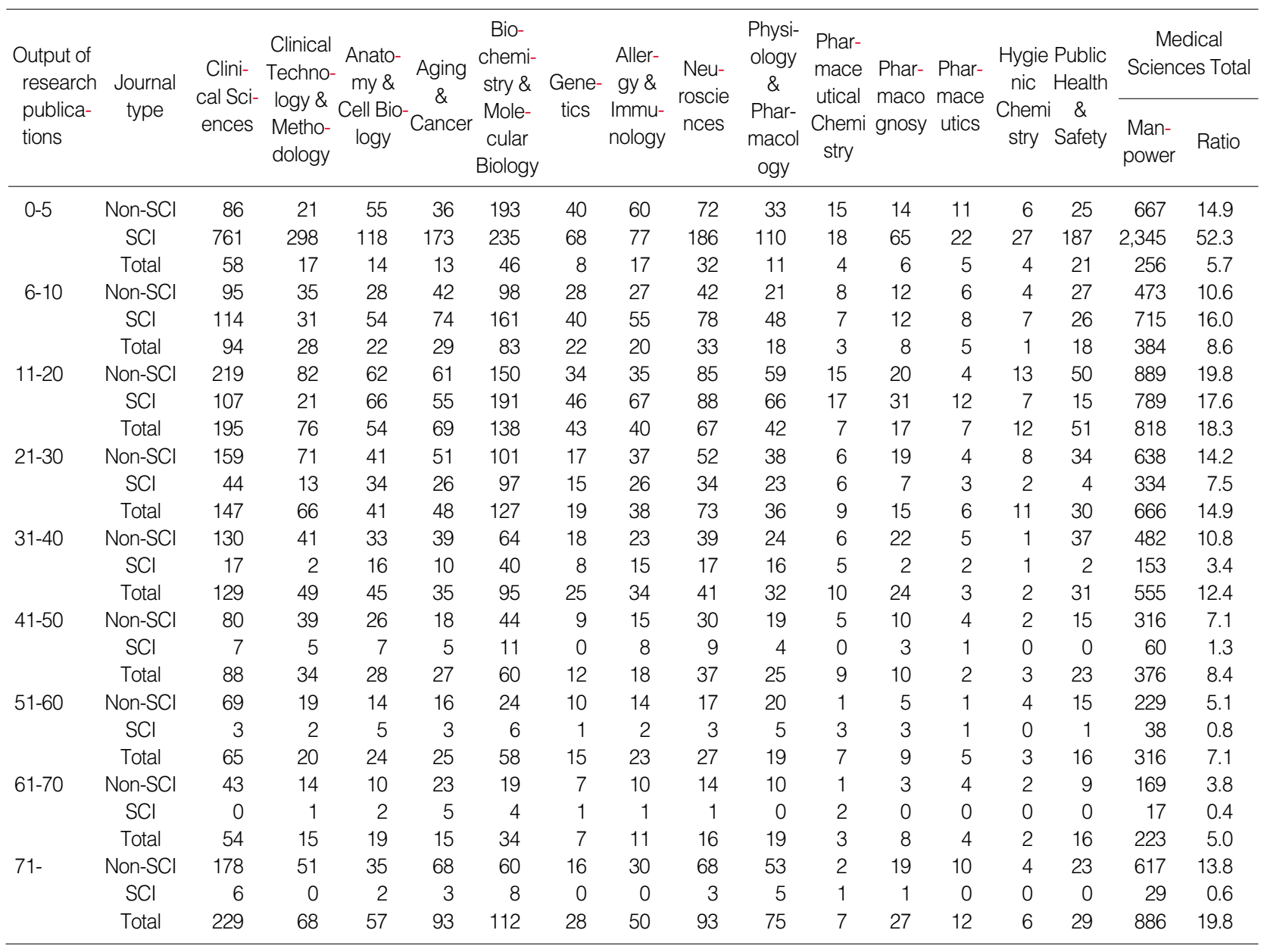

*Source, amended from KOSEF Manpower DB (2004).

sciences and 11.6 million won for other fields (Fig. 3). The average total research expense (29,827 thousand won) per project in the individual-support program in all fields in 2003 was $146 \%$ that of similar funding (Grants for provincial universities, newly-appointed professors and leading researchers) from KRF (8) during the same period. Such a rapid increase in the amount of support per project has played an important role in improving the stability of the research environment for basic science research programs by enhancing the quality and training of competent research manpower.

\section{Analysis of research manpower and number of publica- tions in the medical sciences sector}

KOSEF constructed a research manpower database in the mid 1980s, based on data relating to research grantees and evaluations of project participants, for the systematic management of those who had performed and were capable of performing such research. This system was improved in 2000 by allowing data to be updated by the researchers themselves every two years. Accordingly, it cannot be regarded as the latest data on manpower. Based on the 20,186 researchers who have input their area code out of the total 44,875 registered on the database as of May 2004, the number of research personnel in the medical sciences was 4,495 (22.3\%).

By sector, there are 1,064 researchers $(23.7 \%)$ in the clinical sciences, 377 (8.4\%) in clinical technology \& methodology, $305(6.8 \%)$ in anatomy \& cell biology, $354(7.9 \%)$ in aging \& cancer, 755 (16.8\%) in biochemistry \& molecular biology, $1,778(4.0 \%)$ in genetics, $249(5.5 \%)$ in allergy \& immunology, $417(9.3 \%)$ in neuroscience, $281(6.3 \%)$ in physiology \& pharmacology, $59(1.3 \%)$ in pharmaceutical chemistry, $125(2.8 \%)$ in pharmacognosy, $49(1.1 \%)$ in pharmaceutics, $45(1.0 \%)$ in hygienic chemistry, and $237(5.3 \%)$ in public health \& safety. Among researchers involved in the medical sciences, the youngest average age was $44 \mathrm{yr}$, in biochemistry and molecular biology, allergy and immunology and neuroscience, while the oldest average age, $48 \mathrm{yr}$, was in clinical technology and methodology and pharmaceutical chemistry. Male researchers overwhelmingly dominated with respect to numbers: only $22 \%$ of researchers were female (Table 4 ).

We investigated the number of papers published by KOSEF 
Table 7. Average output of research publications by researchers given grants in medical sciences by program (FYs 2002-2003)

(Unit: Project)

\begin{tabular}{|c|c|c|c|c|c|c|}
\hline Program & Non-SCl & $\mathrm{SCl}$ & Total & $\begin{array}{l}\text { Non-SCI, } \\
\text { recent } 5 \text { yr }\end{array}$ & $\begin{array}{c}\mathrm{SCl}, \\
\text { recent } 5 \mathrm{yr}\end{array}$ & $\begin{array}{l}\text { Total, } \\
\text { recent } 5 \mathrm{yr}\end{array}$ \\
\hline Program for Leading Women Scientists & 33 & 15 & 50 & 20 & 12 & 32 \\
\hline Program for Promising Women Scientists & 17 & 11 & 28 & 13 & 9 & 23 \\
\hline Program for Young Scientists & 15 & 14 & 29 & 12 & 12 & 24 \\
\hline Program for Regional Scientists & 42 & 14 & 59 & 26 & 11 & 38 \\
\hline Program for Leading Scientists & 45 & 27 & 72 & 17 & 21 & 39 \\
\hline Mission-oriented Basic Grants & 48 & 21 & 70 & 26 & 15 & 41 \\
\hline
\end{tabular}

Table 8. Expenditure for academic R\&D in U.S.A.

(Unit: Millions of current dollars)

\begin{tabular}{|c|c|c|c|c|c|c|c|c|}
\hline \multirow{2}{*}{ Field } & \multirow{2}{*}{1975} & \multirow{2}{*}{1980} & \multirow{2}{*}{1985} & \multirow{2}{*}{1990} & \multirow{2}{*}{1995} & \multirow{2}{*}{2000} & \multicolumn{2}{|r|}{2001} \\
\hline & & & & & & & Amount & Distribution, \% \\
\hline Sciences & 3,027 & 5,200 & 8,268 & 13,630 & 18,650 & 25,494 & 27,725 & 84.7 \\
\hline Physical sciences & 350 & 677 & 1,148 & 1,807 & 2,255 & 2,708 & 2,800 & 8.6 \\
\hline Mathematics & 40 & 78 & 128 & 222 & 279 & 341 & 357 & 1.1 \\
\hline Computer sciences & 46 & 124 & 281 & 515 & 682 & 875 & 954 & 2.9 \\
\hline Earth, atmospheric, and ocean sciences & 255 & 508 & 705 & 1,069 & 1,433 & 1,763 & 1,827 & 5.6 \\
\hline Life sciences & 1,900 & 3,218 & 5,279 & 8,725 & 12,186 & 17,460 & 19,190 & 58.6 \\
\hline Agricultural sciences, amount & 384 & 676 & 999 & 1,349 & 1,814 & 2,175 & 2,318 & 7.1 \\
\hline (Agricultural sciences, ratio, \%) & 11.3 & 11.2 & 10.3 & 8.3 & 8.2 & 7.2 & 7.1 & 7.1 \\
\hline Biological sciences & 630 & 1,028 & 1,781 & 2,859 & 3,834 & 5,613 & 5,944 & 18.2 \\
\hline Medical sciences & 811 & 1,423 & 2,318 & 4,154 & 6,070 & 8,986 & 10,177 & 31.1 \\
\hline Other & 75 & 91 & 181 & 363 & 468 & 686 & 751 & 2.3 \\
\hline Psychololgy & 80 & 110 & 158 & 253 & 370 & 516 & 582 & 1.8 \\
\hline Social sciences & 256 & 339 & 383 & 703 & 1,019 & 1,297 & 1,436 & 4.4 \\
\hline Other sciences & 100 & 146 & 186 & 336 & 426 & 534 & 579 & 1.8 \\
\hline Engineering & 381 & 862 & 1,418 & 2,656 & 3,515 & 4,547 & 5,000 & 15.3 \\
\hline Total & 3,408 & 6,062 & 9,686 & 16,286 & 22,165 & 30,041 & 32,725 & 100.0 \\
\hline
\end{tabular}

*Source: National Science Foundation, 2004, Science \& Engineering Indicators 2004, v2. A5-9 A5-11.

researchers in terms of 1 ) the number of papers published in general academic journals not listed in the Science Citation Index (SCI; hereinafter referred to as "number of non-SCI papers"), 2) the number of papers published in SCI academic journals (hereinafter referred to as "number of SCI papers") and 3) total number of papers published including 1) and 2) (hereinafter referred to as "total number of papers"). The nonSCI paper statistics show that among 44,875 researchers, most $(10,025,22.3 \%)$ had published $11-20$ papers, while $8.5 \%$ $(3,804)$ had published more than 70 . For SCI papers, 35,535 researchers $(79.2 \%)$ had published less than 5 papers, and $186(0.4 \%)$ had published more than 70 . In total, the numbers were 11-20 papers (9,942 persons, $22.2 \%)$ ) $>0-5$ papers (7,012 persons, $15.6 \%)$, $>6-10$ papers $(6,900$ persons, $15.4 \%)$, $>21-30$ papers $(6,215$ persons, $13.8 \%)$, > over 70 papers $(5,032$ persons, 11.2\%) (Table 5).

We investigated the publication status of researchers in the medical sciences based on the above statistics. The number of non-SCI papers published was quite uniformly distributed, with 889 researchers $(19.8 \%)$ publishing 11 to 20 papers, followed by $667(14.9 \%)$ who published 0 to 5 papers. For SCI-papers, most $(2,345,52.3 \%)$ were by researchers who published 0-5 papers, while $789(17.6 \%)$ were by researchers who published $11-20$ papers, and only 29 researchers $(0.9 \%)$ published more than 70 papers. Based on the total numbers of papers, the order was as follows: over $70(886,19.8 \%)$, > $11-20(818,18.3 \%),>21-30(666,14.9 \%),>31-40(555,12.4$ $\%),>6-10(384,8.6 \%),>41-50(376,8.4 \%),>51-60(316$, $7.1 \%),>0-5(256,5.7 \%),>61-70(223,5.0 \%)$ (Table 6).

\section{Analysis of papers listed by researchers selected for KOSEF projects}

Researchers selected for Mission-Oriented Basic Grants, which support excellence in small research groups, published an average of 48 papers in non-SCI journals in the most recent 5 -yr period, and averaged 15 papers in SCI journals. The team leaders of Mission-Oriented Basic Grants are outstanding researchers who have led and conducted research for an average of three years, and who have participated actively in the activities of academic societies, in addition to contributing frequently to SCI journals.

Researchers in the program for Leading Scientists, which was implemented to further motivate scientists, foster world- 
leading scientists, and widen Korea's capacity for basic science to a world-class level, published an average of 27 papers in SCI journals, and over the most recent 5-yr period averaged 21 publications in SCI journals. This is the highest number of any SCI sector.

Researchers in the Program for Promising Women Scientists and the program for Young Scientists have published fewer articles in each sector, as compared to other programs. However, since the selected researchers published an average of 28 and 29 papers each in 2002 and 2003, respectively, and in the most recent 5 -yr period averaged a total of 23 and 24 papers each, it is clear that recent performance has improved markedly (Table 7).

\section{Analysis of research funding in the major advanced coun- tries}

Analysis of academic funding in the USA shows that of a total of 32.7 billion dollars (2001 basis), $31.1 \%$ or 2.3 billion dollars were allocated to the medical sciences and $58.6 \%$ or 19.2 billion dollars were allocated to all the life sciences, including agricultural science, biology, and others. The increasing trend, evident in annual figures for the medical sciences of 0.811 billion dollars in 1975, 2.318 billion dollars in 1985, 4.154 billion dollars in 1990 and 10.177 billion dollars in 2001 , roughly corresponds to the case analyzed in this paper (Table 8).

\section{DISCUSSION}

From an economic applications perspective, derived from basic research, it has been reported that government investment in basic research provides returns to society and makes a crucial contribution to economic growth. Moreover, a percentage of the increase in sales by companies results from taking advantage of university research. Basic research provides society with practical information, new forms of mechanization and methodologies, trained manpower, a systematic organizational structure based on expertise and technological solutions to problems, and leads to the creation of new companies (9).

As a result of the importance of basic research, research spending has been the fastest growing item in the national budget. However, the medical sciences will inevitably require longer-term and more stable investment than is offered to other fields. Stable opportunities for academic and research jobs, in comparison with other fields, will prevent the brain drain of promising new researchers and graduates. It will be necessary to establish a plan to strengthen our research competitiveness in order to entice our brightest minds to return to Korea and occupy positions of leadership in society, as well as in research institutes. This may be effected, in part, by developing new inter-disciplinary studies in the life sciences. In addition, we must acknowledge the external threats created by grouping specific university graduates.

In this study we analyzed both the strengths and weaknesses of the internal environment for research in the medical sciences, as well as the opportunities offered and threats posed by the external environment surrounding the medical sciences, in relation to other academic fields. As compared to other fields, the major strengths of the medical sciences lies in their research manpower, the advanced level of their research facilities, and research and technological capabilities that are not far behind those of the most advanced countries.

Concerning weaknesses in this area, first, relatively little attention has been paid to strengthening relationships between different academic fields or to generating new inter-disciplinary areas; this is because the relevant societies and research institutes have failed to assume a leadership role in this respect. Second, researchers' preferences for the medical sciences have led to limited research manpower being available for other fields. Third, the distribution of research endeavor on the part of universities has not diversified and has, instead, been grouped according to specific university background. Fourth, few government officials, such as ministers and vice-ministers, are qualified to take part in the decision-making process in this field, as compared to other areas, such as the social sciences, physics, chemistry, agriculture and fisheries, and engineering. Furthermore, opportunities for these officials to do so have been considerably limited.

As for the opportunities offered by the external environment, increasing emphasis has been placed on medical research and there has been increased interest in biotechnology. In addition, many international cooperative research projects are presently underway, thanks to the relatively small gap in capabilities, even in relation to the most advanced countries. Finally, the biotechnology industry is still at a primitive stage and many areas in this field remain to be explored.

In contrast, the external environment can pose a threat in that creative and stable long-term research activities pertaining to basic research in the medical sciences, with relatively long recovery periods, have not been implemented smoothly by the investment of research funds in such visible areas as the 10 Growth Propulsion Power and The National Technology Guidance projects.

\section{Research trends in the medical sciences by the type of programme}

Around 1990, many large-scale research programs emerged owing to the influence of research in advanced countries. These started with the awarding of group Mission-Oriented Basic Grants, which gave rise to SRC/ERC in February 1990 and inter-disciplinary cooperative research projects. Subsequently, the proportion of funds supporting individual projects and manpower training programs has increased gradually. Support for research in the medical sciences has improved steadily 
since 1986, thanks to the Research Support Project for Specialization Encouragement, which was initiated in 1995.

For the sciences in general, support for group research versus individual research has grown rapidly since 2000. With this increase in group research has come an increased awareness of the benefits of inter-disciplinary co-operative research. Programs supporting group research, such as SRC/ERC, Regional Research Center, Medical Research Center, and local university-based research support, play a significant role in enhancing the national level of capability with respect to science technology. For these projects to be successful there must be a sound framework that allows individual researchers at universities and institutes to conduct creative, ongoing projects. Fundamental research in various fields is necessary to meet the needs of a rapidly changing society, and to boost its capability to incorporate future technology. As support has tended to focus on group research, it has become more difficult for individual researchers not involved in groups to undertake research. For this reason, we need to construct a stronger base for basic research.

Comparing the rate of successful applications to the National Science Foundation of the United States (NSF) and the KRF, in 2003, the NSF awarded 10,844 grants in response to a total of 40,075 applications (27\%), while in 2003 the KRF awarded 2,433 grants in response to 9,523 applicants (23\%). For specific programs sponsored by the KOSEF, the success rate was even lower: $11 \%$ for programs supporting research scientists (63 out of 589), $11 \%$ for those fostering leading scientists (25 out of 176), and $22 \%$ for the program promoting excellence among scientists in regional universities (361 out of 1,656). Furthermore, the rate of support for new projects has decreased each year since 2002: it was 39\% in 2002 (932 of 2,380), 26\% in 2003 (1,002 of 3,844), and $20 \%$ in 2004 (738 of 3,867$)$. In this regard, inter-departmental competition for group research program grants has been severe. Consequently, the funds available to individuals have been greatly limited, and the support for young scientists is decreasing. Therefore, when designing group research programs, it is necessary to establish a solid national support program, as a part of the National Millennium Plan, through extensive evaluation of the expertise available, after considering the opinions of scientists.

In part, the lower proportion of grants for individual research programs, compared to group research programs in Table 3, occurred because the average unit research cost of group research is considerably higher than that of other programs. More attention should be paid not only to increasing the unit cost, but also to enlarging support for individuals, infrastructure, and manpower training programs in order to improve the research environment.

There was considerable support for individual programs up to the late 1980s. Beginning in the 1990s, grants favored group research or center-supported programs, and this trend continued until 2003. The reason for this decline in support for individual programs was that group research had an advantage in terms of increased output of publications, both qualitatively and quantitatively $(10,11)$, while fewer research activities were available to researchers who were not invited into large-scale projects. However, even among individual projects, it is not easy to conclude that giving large amounts of money to a few projects increases output, as compared to giving small amounts to numerous projects. Moreover, it has been suggested that group research does not result in output of a quality that is equivalent to that from individuals $(10,11)$. A slight increase in the rate of grants to individual projects $(25.1 \%)$ in 2003 might be regarded as an appropriate measure to counter these problems.

Our research support system can be described as an inverted triangle. There was a severe imbalance in the amounts granted in 2001, as only $11 \%$ was invested in infrastructure supporting individual and group/collective projects, which were deemed a focus, whereas $37 \%$ went to specific research areas, and $52 \%$ was poured into special tasks that were initiated nationally. Researchers in charge of infra-research accounted for $15 \%$ of the researchers receiving grants. High-level researchers at SRCs/ERCs accounted for $40 \%$ (10). Theoretically, the ideal situation occurs when at least $70 \%$ goes to individual-oriented tasks, $20-30 \%$ to collective research in specific areas by medium-sized groups, with the balance going to special nationally designated large-scale research projects. In the U.S.A. in 2002, most of the university R\&D funds, about $74 \%$, were invested in basic research comprised mainly of projects undertaken by individuals, whereas applied research received $22 \%$ and $\mathrm{R} \& \mathrm{D} 4 \%$ (7)

From this perspective, it appears highly advisable to review policy on national $\mathrm{R} \& \mathrm{D}$ projects and research budget allocation. Conversely, coverage by the manpower-training program increased between 1995 and 1999, but then decreased following the discontinuance of the domestic post-doc training program and the curtailment of foreign post-doc training programs as a result of the Korean financial crisis in 1997. Certain measures are needed to enhance the quality of the next generation of researchers in the medical sciences.

\section{Unit cost trends in the medical sciences}

Research funding per project has increased every year, as seen in Table 4, thanks to the implementation of MissionOriented Basic Grants in 1987 and the SRC/ERC program in 1990. Although less than the NSF average of $\$ 135,609$ per research project in 2003 (4), it is much more than the sum of approximately 47 million won that was provided by the Korea Research Foundation under the MOE and Human Resources Development (8), and it can be regarded as an appropriate amount in terms of the average amount vs. the per capita gross national product (GNP). The report by the MOE stating that the average $\mathrm{R} \& \mathrm{D}$ expense of 40 million won per university staff in 2003 was $25 \%$ (8 million won) higher 
than two years ago is further evidence of the improvement.

\section{Number of publications in the medical sciences sector}

It is noteworthy that among researchers publishing more than 70 non-SCI papers, the percentage in the medical sciences $(13.8 \%)$ is far higher than in other fields (average: $8.5 \%$; Table 7), as is also the case for the total number of papers published (19.8\%). Researchers in the medical sciences published numerous papers in domestic academic journals, and these publications were derived from a more solid research framework than has often been the case previously. In the past, scant attention was paid to the SCI academic journals. The publications in the medical sciences need further consideration in terms, simultaneously, of both their qualitative and quantitative aspects.

\section{CONCLUSIONS AND SUGGESTIONS}

The numbers show that in the medical sciences, more investment has been made in group/collective research than in projects undertaken by individuals, and that the level of funding per unit project has been a little lower than the overall amount in engineering fields. According to the statistics for grants and the numbers of projects supported, medical research expenditure has been the fastest-growing item. The numbers of papers published in the last five years, according to funding, were in the following order: Mission-Oriented Basic Grants> Program for Leading Scientists $>$ Program for Regional Scientists $>$ Program for Leading Women Scientists $>$ Program for Young Scientists $>$ Program for Promising Women Scientists.

Research in the medical sciences is an essential enterprise leading to the benefit of our society. It is undertaken, to a very large extent, in laboratories scattered throughout academic health centers by dedicated, expert scientists who are driven by a quest to help improve the quality of life. Both investment and the numbers of research programs must be increased. Furthermore, we need to take appropriate measures to activate research activities. Finally the medical sciences should maintain their share of manpower, resources and research funds, in competition with other fields of science and engineering. To do so, we need to participate aggressively in political debate. In order to overcome intrinsic weaknesses, we need to seize available opportunities, encourage positive research activities, and utilize our strengths. In the future, as the knowledge-based society evolves, medical science research will play a leading role in biotechnology; hence, the policy measures described above should be implemented. Although funding of medical science research is much lower in Korea than in the most advanced countries, such research activities can be of value to related industries in that they can facilitate the realization of policy measures, the effective execution and control of research budgets, and the ambitions of researchers.

The ongoing revolution in the medical sciences is of an unprecedented magnitude. Furthermore, it is accelerating dramatically, and it promises almost unlimited opportunity for the betterment of mankind. Despite potential obstacles and unforeseen challenges, the possibilities for progress in the medical sciences and the opportunities for medical research have never been greater. For progress in medical research to continue, sustained and sufficient funding to support the research infrastructure and to encourage innovation is necessary. Indeed, it has been shown that national investment in medical research has produced "outstandingly high returns" in the past and that sustained investment in biomedical research is likely to continue to deliver high returns, not simply by reducing mortality, but more importantly by extending healthy life.

\section{REFERENCES}

1. Jang JK. Performance analysis of investment in basic research. KOSEF, Daejon, Korea 2002; 4-5.

2. Office for Official Publications of the European Community (OECD). Third European Report on Science and Technology Indicators, 2003; 96.

3. Min TS, Choi HK, Kim SY, Bai SC, Kim YY, Yang MS, Chung BH, Hwang JY, Han IK. A proposal for promotion of research activities by analysis of KOSEF's basic research supports in agricultural sciences. J Korean Soc Appl Biol Chem 2005; 48: 23-33.

4. National Science Foundation (NSF). Science \& engineering indicators 2004, 2004; 1: 0-1 0-19, 2: A5-9 A5-11, A3-16.

5. Korea Institute of S\&T Evaluation and Planning (KISTEP). National R\&D Expenditures in 2003, KISTEP, Seoul, Korea 2003; 63-7.

6. Congress of Deans of Medical Schools in National Universities (CDM$\mathrm{SNU})$. Special Report on the Development of Medical Science in the 21st Century, CDMSNU, Seoul, Korea 2000; 37-185.

7. Korea Science and Engineering Foundation (KOSEF). KOSEF-supported research: citation analysis (1998-2002), KOSEF, Daejon, Korea 2004; 14-6.

8. Korea Research Foundation (KRF). Annual statistics on research grants in 2004, KRF, Seoul, Korea 2004; 65-146.

9. Nature. Basic research: an economic good, Nature 382, 1996; 741.

10. Korea Science and Engineering Foundation (KOSEF). KOSEF Statistics 2003, KOSEF, Daejon, Korea 2003a; 317.

11. Korea Science and Engineering Foundation (KOSEF). Report of research output on KOSEF activities 2003, KOSEF, Daejon, Korea $2003 b ; 250$. 